\title{
Vibratory diagnosis by finite element model updating and operational modal analysis
}

\author{
Guillaume Gautier ${ }^{a}$, Roger Serra and Jean-Mathieu Mencik
}

ENI Val de Loire, Université François Rabelais de Tours, LMR Laboratory, Rue de la Chocolaterie, BP 3410, 41034 Blois Cedex, France

\author{
Received 30 November 2012, Accepted 11 April 2013
}

\begin{abstract}
In this paper, a subspace fitting method is proposed to update, in the time domain, the finite element model of a rotating machine. The procedure is achieved by minimizing an error norm, leading to the comparison between experimental and theoretical observability matrices. Experimental observability matrix is obtained through a MOESP subspace identification algorithm, by projecting the output signal onto some appropriate subspaces, resulting in a cancellation of input excitations and noises. The theoretical observability matrix is obtained from modal parameters of a finite element model of the structure. The minimization procedure is carried out through a Gauss-Newton algorithm. The method is applied to determine the foundation stiffness of an experimental rotating machine subject to a random noise.
\end{abstract}

Key words: Subspace fitting / operational modal analysis / finite elements model updating / vibratory diagnosis / rotating machine

\section{Nomenclature}

\begin{tabular}{|ll|}
\hline$\Omega$ & Angular velocity $\left(\mathrm{rad} . \mathrm{s}^{-1}\right)$ \\
$\rho$ & Density $\left(\mathrm{kg} \cdot \mathrm{m}^{-3}\right)$ \\
$E$ & Young modulus of the shaft $\left(\mathrm{N} \cdot \mathrm{m}^{-2}\right)$ \\
$G$ & Shear modulus of the shaft $\left(\mathrm{N} \cdot \mathrm{m}^{-2}\right)$ \\
$I$ & Inertia moment of the shaft $\left(\mathrm{kg} \cdot \mathrm{m}^{2}\right)$ \\
$I_{\mathrm{D} y, z}$ & Inertia moments of the disk $\left(\mathrm{kg} \cdot \mathrm{m}^{2}\right)$ \\
$k_{\mathrm{B}}$ & Bearing stiffness $\left(\mathrm{N} \cdot \mathrm{m}^{-1}\right)$ \\
$L$ & Length of the shaft $(\mathrm{m})$ \\
$m_{\mathrm{D}}$ & Mass of the disk $(\mathrm{kg})$ \\
$S$ & Cross-sectional area of the shaft $\left(\mathrm{m}^{2}\right)$ \\
\hline
\end{tabular}

\section{Introduction}

Evaluating damages occurring in mechanical systems constitutes a tough task. Their emergence and evolution are characterized by variations (those can be small) of the dynamic properties of structures [1]. Many damage diagnosis methods have been proposed to carry out this issue. The methods based on Finite Element (FE)

\footnotetext{
${ }^{a}$ Corresponding author:

guillaume.gautier@etu.univ-tours.fr
}

model updating [2] perform the comparison between the modal parameters obtained experimentally with those of a numerical model. For industrial processes, Operational Modal Analysis (OMA) [3] approaches aim at extracting the structural parameters in operating conditions. Among these approaches, Subspace Identification (SubID) techniques [4-6] appear highly efficient to determine the modal parameters of structures in the time domain. The framework of SI techniques is summarized as follows. From the consideration of input-output data, a so-called experimental observability matrix is obtained by projecting the output signal onto some appropriate subspaces. The observability matrix contains the modal parameters of the structure considered, which are extracted using a subspace fitting method $[7,8]$. The motivation behind this work is to improve further on the accuracy of SI techniques to predict the modal parameters of any mechanical system. To this aim, a FE model of the structure is considered to carry out the subspace fitting procedure in a Least Squares (LS) sense. The modal parameters of the system are then updated by minimizing an error norm which depends on some unknown parameters of the structure (e.g., stiffnesses...). The proposed approach is applied to a rotating machine excited by a random noise. The experimental observability matrix, as obtained using the MOESP SubID technique, is used to update the parameters of the structure. The accuracy of the method is highlighted. 


\section{Deterministic-stochastic modal analysis}

The purpose of SI techniques [5] is to consider a discrete modal state-space representation of the form

$$
\begin{aligned}
\boldsymbol{q}_{k+1} & =\boldsymbol{\Lambda} \boldsymbol{q}_{k}+\boldsymbol{B}_{\bmod } \boldsymbol{u}_{k}+\boldsymbol{w}_{k} \\
\boldsymbol{y}_{k} & =\boldsymbol{\Phi}_{\mathrm{obs}} \boldsymbol{q}_{k}+\boldsymbol{v}_{k}
\end{aligned}
$$

where $\boldsymbol{u}_{k}$ and $\boldsymbol{y}_{k}$ are the vectors of input and output data, respectively; $\boldsymbol{\Lambda}$ is a diagonal matrix of eigenvalues; $\boldsymbol{B}_{\text {mod }}$ and $\boldsymbol{\Phi}_{\text {obs }}$ are matrices expressed in terms of the mode shapes of the structure; also, $\boldsymbol{w}_{k}$ and $\boldsymbol{v}_{k}$ are vectors of noises while $\boldsymbol{q}_{k}$ is a vector of generalized coordinates. In (1), the subscripts $k$ and $k+1$ refer to vectors of data measured at two different times $t_{k}$ and $t_{k+1}=t_{k}+\Delta t$. From the linear state space model, an input-output matrix equation is derived as [6]

$$
\boldsymbol{Y}=\boldsymbol{\Gamma}_{\exp } \boldsymbol{Q}+\boldsymbol{H}^{d} \boldsymbol{U}+\boldsymbol{H}^{s} \boldsymbol{R}+\boldsymbol{N}
$$

where $\boldsymbol{Y}$ is a matrix of output data that are measured over different time intervals $\left\{\left[t_{k} t_{k+1} \ldots t_{k+\alpha-1}\right]\right\}_{k} . \boldsymbol{Q}, \boldsymbol{H} \boldsymbol{R}$ and $\boldsymbol{N}$ are the related matrices of generalized coordinates, input data and noises; also, $\boldsymbol{\Gamma}_{\exp }$ represents the experimental observability matrix; otherwise, $\boldsymbol{H}^{d}$ and $\boldsymbol{H}^{s}$ are Hankel matrices. The basic idea behind SubID techniques is to identify the experimental observability matrix $\boldsymbol{\Gamma}_{\exp }$ from the knowledge of $\boldsymbol{Y}$. This is done by eliminating the terms $\boldsymbol{H}^{d} \boldsymbol{U}+\boldsymbol{H}^{s} \boldsymbol{R}+\boldsymbol{N}$ in (3) by means of projection and weighting procedures. Clearly, a projection of the row space of $\boldsymbol{Y}$ onto the orthogonal complement $\boldsymbol{U}^{\perp}$ of the row space of $\boldsymbol{U}$ enables one to remove the influence of inputs. In addition, the fact to left and right multiply (3) with some matrices $\boldsymbol{W}_{1}$ and $\boldsymbol{W}_{2}$ having some specific properties regarding noise uncorrelation enables those noise terms to be removed as well. Considering such procedures yields

$$
\boldsymbol{O}=\boldsymbol{W}_{1} \boldsymbol{Y} / \boldsymbol{U}^{\perp} \boldsymbol{W}_{2}=\boldsymbol{W}_{1} \boldsymbol{\Gamma}_{\exp } \boldsymbol{Q} / \boldsymbol{U}^{\perp} \boldsymbol{W}_{2}
$$

More specifically, SI techniques deal with the matrix $\boldsymbol{W}_{1} \boldsymbol{\Gamma}_{\exp }=\boldsymbol{U}_{1} \boldsymbol{S}_{1}^{1 / 2}[5]$, where the matrix $\boldsymbol{S}_{1}$ results from a SVD of $\boldsymbol{O}$, i.e.

$$
\boldsymbol{O}=\left[\begin{array}{ll}
\boldsymbol{U}_{1} & \boldsymbol{U}_{2}
\end{array}\right]\left[\begin{array}{cc}
\boldsymbol{S}_{1} & \mathbf{0} \\
\mathbf{0} & \simeq \mathbf{0}
\end{array}\right]\left[\begin{array}{c}
\boldsymbol{V}_{1}^{T} \\
\boldsymbol{V}_{2}^{T}
\end{array}\right]
$$

The matrices of eigenvalues $\boldsymbol{\Lambda}$ and mode shapes $\boldsymbol{\Phi}_{\text {obs }}$ are determined from the experimental observability matrix $\boldsymbol{\Gamma}_{\exp }$ in different ways [6]. All the methods make use of the invariance property of the matrix $\boldsymbol{\Gamma}_{\exp }$.

\section{Subspace fitting for finite element model updating}

\subsection{Objective function}

The subspace fitting procedure $[7,8]$ is a concept that aims at correlating a theoretical matrix $\boldsymbol{\Gamma}(\boldsymbol{\theta})$ with the experimental observability matrix $\boldsymbol{\Gamma}_{\exp }$ as

$$
\boldsymbol{\Gamma}_{\exp }=\boldsymbol{\Gamma}(\boldsymbol{\theta}) \boldsymbol{T}
$$

where $\boldsymbol{T}$ is a similarity matrix. Here, the matrix $\boldsymbol{\Gamma}(\theta)$ is supposed to be dependent from a set of parameters (denoted as $\boldsymbol{\theta}$ ) which are to be identified. The subspace fitting procedure can be formulated through the following LS problem:

$$
\{\boldsymbol{\theta}, \boldsymbol{T}\}=\operatorname{argmin}\left\|\boldsymbol{\Gamma}_{\exp }-\boldsymbol{\Gamma}(\boldsymbol{\theta}) \boldsymbol{T}\right\|_{F}^{2}
$$

where $\|.\|_{F}$ denotes the Frobenius norm. This LS problem can be simplified by determining the matrix $\boldsymbol{T}$ in a preprocessing step as $\boldsymbol{T}=\boldsymbol{\Gamma}(\boldsymbol{\theta})^{+} \boldsymbol{\Gamma}_{\exp }\left(\boldsymbol{\Gamma}(\boldsymbol{\theta})^{+}\right.$being the pseudo-inverse of $\boldsymbol{\Gamma}(\boldsymbol{\theta})$ ), which yields

$$
\boldsymbol{\theta}=\operatorname{argmin}\|\boldsymbol{r}(\boldsymbol{\theta})\|_{2}^{2}
$$

where $\boldsymbol{r}(\boldsymbol{\theta})=\operatorname{vec}\left\{\left(\boldsymbol{I}-\boldsymbol{\Gamma}(\boldsymbol{\theta}) \boldsymbol{\Gamma}(\boldsymbol{\theta})^{+}\right) \boldsymbol{\Gamma}_{\exp }\right\}$.

The key idea behind the present work is to express the theoretical observability matrix $\boldsymbol{\Gamma}(\boldsymbol{\theta})$ by means of a FE model of the considered mechanical system. In doing so, the spatial dynamics of the system is taken into account to carry out the minimization procedure of $\boldsymbol{r}(\boldsymbol{\theta})$ with a view to identifying the parameters $\boldsymbol{\theta}$ in an accurate and unique way.

Regarding rotating machines, a related FE based eigenproblem is considered as

$$
\left(\mu_{j}^{2} \boldsymbol{M}+\mu_{j}(\gamma+\Omega \boldsymbol{G})+\boldsymbol{K}\right) \boldsymbol{\Phi}_{j}=0
$$

where $\boldsymbol{M}, \boldsymbol{K}$ and $\gamma$ refer to the mass, stiffness and damping matrices; also, $\boldsymbol{G}$ is the matrix that reflects the gyroscopic effects. The solutions of the eigenproblem are $\left\{\mu_{j}, \boldsymbol{\Phi}_{j}\right\}$ which stand for complex eigenvalues and right eigenvectors, respectively. Thus a theoretical FE-based observability matrix can be expressed as

$$
\boldsymbol{\Gamma}(\boldsymbol{\theta})=\left[\begin{array}{c}
\boldsymbol{\Phi}_{\mathrm{obs}} \\
\boldsymbol{\Phi}_{\mathrm{obs}} \boldsymbol{\Lambda} \\
\vdots \\
\boldsymbol{\Phi}_{\mathrm{obs}} \boldsymbol{\Lambda}^{\alpha-1}
\end{array}\right]
$$

where $\boldsymbol{\Lambda}=\mathrm{e}^{\Delta t \operatorname{diag}\left(\mu_{i}\right)}$ and $\boldsymbol{\Phi}_{\text {obs }}$ is the matrix of eigenvectors $\left\{\boldsymbol{\Phi}_{j}\right\}$ at the observation points of output signals.

\subsection{Optimization algorithm}

The Gauss-Newton algorithm [9] is used to solve the minimization problem (9). This algorithm is based on the following iterative scheme

$$
\boldsymbol{\theta}_{f+1}=\boldsymbol{\theta}_{f}-\beta_{f} \boldsymbol{H}^{-1} \boldsymbol{g}
$$

where $\beta_{f}$ is a step size, while $\boldsymbol{g}$ and $\boldsymbol{H}$ are Gradient and Hessian matrices of $\|r(\boldsymbol{\theta})\|_{2}^{2}$, defined as

$$
\boldsymbol{g}_{i}=2 \operatorname{Re}\left\{\boldsymbol{r}^{H} \frac{\partial \boldsymbol{r}}{\partial \theta_{i}}\right\}
$$

and

$$
\boldsymbol{H}_{i j}=2 \operatorname{Re}\left\{\frac{\partial \boldsymbol{r}^{H}}{\partial \theta_{i}} \frac{\partial \boldsymbol{r}}{\partial \theta_{j}}\right\}
$$




$$
\boldsymbol{K}_{\mathrm{S}}=\frac{E I}{(1+a) L^{3}}\left[\begin{array}{ccccccccc}
12 & 0 & 0 & 6 L & -12 & 0 & 0 & 6 L \\
& 12 & -6 L & 0 & 0 & -12 & -6 L & 0 \\
& & 4 L^{2}+a & 0 & 0 & 6 L & 2 L^{2}-a & 0 \\
& & & 4 L^{2}+a & -6 L & 0 & 0 & 2 L^{2}-a \\
& & & & & 12 & 0 & 0 & -6 L^{2} \\
& & & & & & & & \\
& & & & & & 4 L^{2}+a & 0 \\
& & & & & & & & 4 L^{2}+a
\end{array}\right]
$$

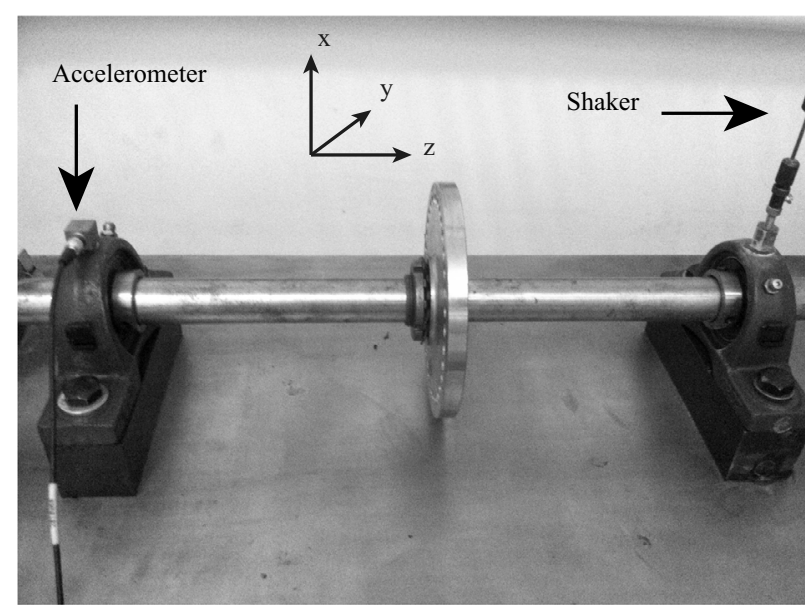

Fig. 1. Experimental structure.

Table 1. Properties of the structure.

\begin{tabular}{ccc}
\hline & Geometrical proprieties & Physical proprieties \\
\hline \multirow{3}{*}{ Shaft } & Length $=0.50 \mathrm{~m}$ & $E=193 \times 10^{9} \mathrm{~N} \cdot \mathrm{m}^{-2}$ \\
& Diameter $=0.0254 \mathrm{~m}$ & $G=74.2 \times 10^{9} \mathrm{~N} \cdot \mathrm{m}^{-2}$ \\
& & $\rho=7818 \mathrm{~kg} \cdot \mathrm{m}^{-3}$ \\
Disk & Thickness $=0.01 \mathrm{~m}$ & $\rho=7818 \mathrm{~kg} \cdot \mathrm{m}^{-3}$ \\
& Diameter $=0.13 \mathrm{~m}$ & \\
\hline
\end{tabular}

\section{Experiments}

\subsection{Description of the structure}

The proposed method is applied to update the FE model of the rotating machine depicted in Figure 1. The structure is composed of a shaft attached to one rigid disk and supported by two flexible bearings. The properties of the structure are reported in Table 1 . The output signal of the structure is measured by means of one accelerometer which is attached to the first bearing. On the second bearing, a shaker generates a random noise. The experimental observability matrix is obtained using the MOESP SubID technique [6]. Some of identified experimental eigenfrequencies are reported in Table 2.

\subsection{FE model of the structure}

A FE model is considered which is composed of six Timoshenko beam elements for the shaft, with two translations ( $u$ and $v$ ) and two rotations $(\psi$ and $\phi)$ per node, along the $x$ and $y$-axis.

The nodal displacement vector is denoted as

$$
\boldsymbol{\delta}=\{u v \phi \psi\}^{T}
$$

The mass matrices $\boldsymbol{M}_{\mathrm{S}}$ and $\boldsymbol{M}_{\mathrm{S}}^{r}$ of a beam element, related to translational and rotational displacements, are respectively expressed as

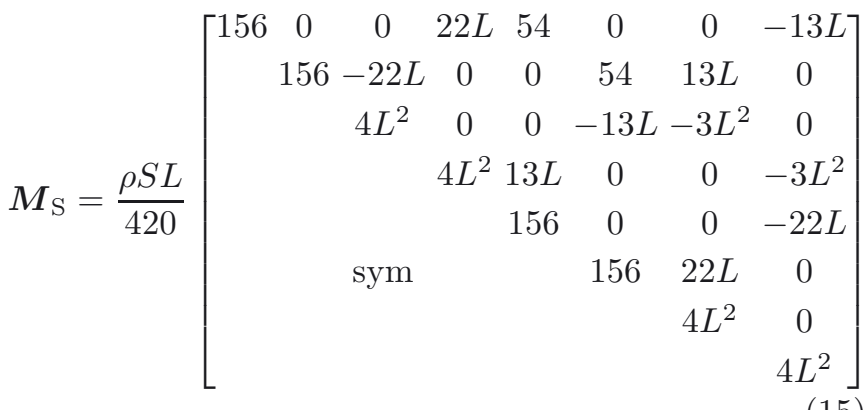

and

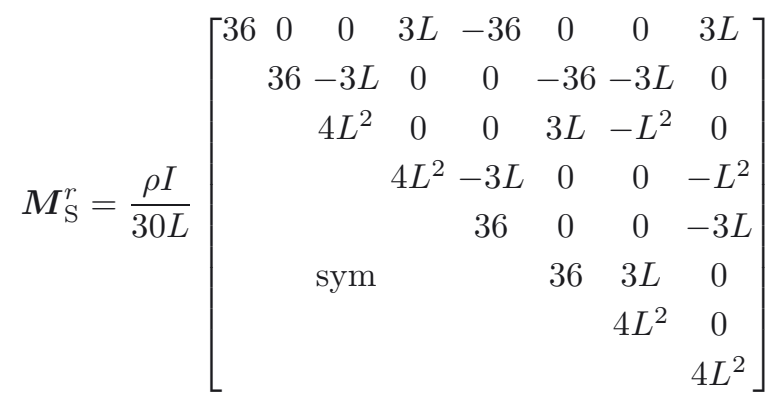

Also, the related matrix of gyroscopic effects is given by

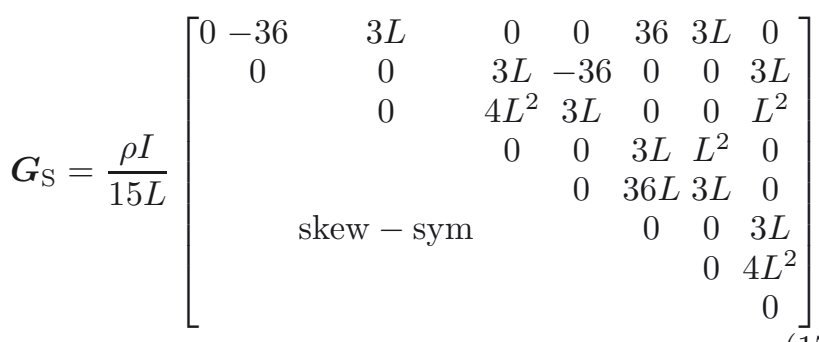

The element stiffness matrix is given by

$$
\text { see equation (18) above }
$$

where $a=\frac{12 E I}{G S L^{2}}$ is introduced to take into account the shear deformation effects. Finally, the whole equation of 
Table 2. Eigenfrequencies of the system (rotating machine - foundation).

\begin{tabular}{cccccc}
\hline & $\begin{array}{c}\text { Experimental } \\
\text { frequency (Hz) }\end{array}$ & \multicolumn{2}{c}{ Before updating } & \multicolumn{2}{c}{ After updating } \\
\cline { 3 - 6 } & 631 & 394.7 & 37 & 635.8 & 0.8 \\
\hline 1 & 884 & 759.5 & 14.1 & 878.9 & 0.6 \\
2 & 1551 & 1294.3 & 16.6 & 1552.1 & 0.1 \\
3 & 2399 & 2064.6 & 13.9 & 2284.9 & 4.8 \\
4 & 3001 & 2606.1 & 13.2 & 2962.9 & 1.3 \\
5 & \multicolumn{3}{c}{} \\
\hline
\end{tabular}

motion for the shaft element is expressed as

$$
\left(\boldsymbol{M}_{\mathrm{S}}+\boldsymbol{M}_{\mathrm{S}}^{r}\right)\left\{\begin{array}{l}
\ddot{\boldsymbol{\delta}_{1}} \\
\ddot{\boldsymbol{\delta}_{2}}
\end{array}\right\}+\Omega \boldsymbol{G}_{\mathrm{S}}\left\{\begin{array}{l}
\dot{\boldsymbol{\delta}_{1}} \\
\dot{\boldsymbol{\delta}_{2}}
\end{array}\right\}+\boldsymbol{K}_{\mathrm{S}}\left\{\begin{array}{l}
\boldsymbol{\delta}_{1} \\
\boldsymbol{\delta}_{2}
\end{array}\right\}=0
$$

where structural damping is neglected.

Otherwise, the disk is modeled by means of concentrated mass and gyroscopic effects using the following matrix term:

$$
\begin{aligned}
\boldsymbol{M}_{\mathrm{D}} \ddot{\boldsymbol{\delta}}+\Omega \boldsymbol{G}_{\mathrm{D}} \dot{\boldsymbol{\delta}}= & {\left[\begin{array}{cccc}
m_{\mathrm{D}} & 0 & 0 & 0 \\
0 & m_{\mathrm{D}} & 0 & 0 \\
0 & 0 & I_{\mathrm{D} x} & 0 \\
0 & 0 & 0 & I_{\mathrm{D} x}
\end{array}\right]\left[\begin{array}{c}
\ddot{u} \\
\ddot{v} \\
\ddot{\psi} \\
\ddot{\phi}
\end{array}\right] } \\
& +\Omega\left[\begin{array}{cccc}
0 & 0 & 0 & 0 \\
0 & 0 & 0 & 0 \\
0 & 0 & 0 & -I_{\mathrm{D} z} \\
0 & 0 & I_{\mathrm{D} z} & 0
\end{array}\right]\left[\begin{array}{c}
\dot{u} \\
\dot{v} \\
\dot{\psi} \\
\dot{\phi}
\end{array}\right]
\end{aligned}
$$

Finally, the foundation of the bearings is modeled by means of the following stiffness matrix

$$
\boldsymbol{K}_{\mathrm{B}}=\left[\begin{array}{cccc}
k_{\mathrm{B}} & 0 & 0 & 0 \\
0 & k_{\mathrm{B}} & 0 & 0 \\
0 & 0 & 0 & 0 \\
0 & 0 & 0 & 0
\end{array}\right]
$$

\subsection{Updating procedure}

The updating procedure of the FE model is carried out considering the bearing stiffness $k_{\mathrm{B}}$ as an unknown parameter. This parameter is updated through the subspace fitting procedure described previously, whose flowchart is postponed in Figure 2.

The procedure is initialized with a value of $10 \times$ $10^{6} \mathrm{~N} . \mathrm{m}^{-1}$ for $k_{\mathrm{B}}$. The eigenfrequencies of the system (rotating machine - foundation), obtained for this value, are reported in Table 2 and compared with the experimental eigenfrequencies. Thus the updating procedure is carried out which yields a value of $33 \times 10^{6} \mathrm{~N}^{-m^{-1}}$ for $k_{\mathrm{B}}$. In that case, the errors between FE-based and experimental eigenfrequencies appear quite small, as expected. In a more general view, the updating procedure can be used to determine the variation of the stiffness parameter $k_{\mathrm{B}}$ over the time domain to carry out structural health monitoring. This yields an efficient way to detect the occurrence of defects as well as machine breakdowns.

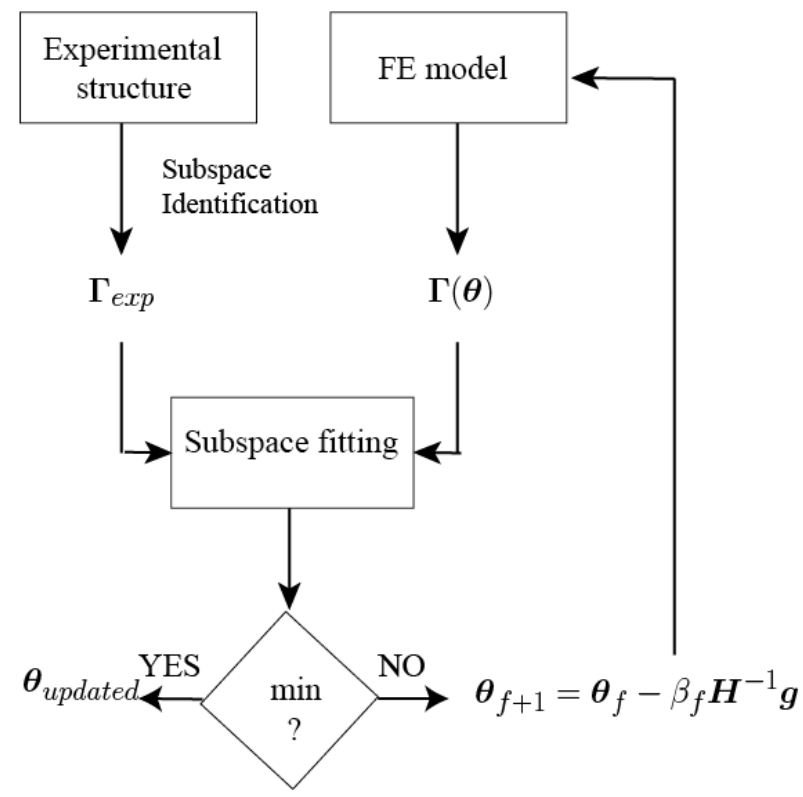

Fig. 2. Flowchart of the subspace fitting procedure.

\section{Conclusion}

A finite element model updating procedure has been proposed. The procedure used a subspace fitting approach to adjust a FE-based observability matrix with an experimental observability matrix obtained from a subspace identification technique. The method has been successfully applied to determine the bearing stiffness of a rotating machine. In a more general view, this method seems to constitute an efficient tool to carry out the structural health monitoring of mechanical systems.

Acknowledgements. The authors express their thanks for the financial support provided by European Union (FEDER Centre) and Conseil Régional du Centre (France).

\section{References}

[1] S.W. Doebling, C.R. Farrar, M.B. Prime, A summary review of vibration-based damage identification methods, Shock Vibr. Dig. 30 (1998) 91-105

[2] J.E. Mottershead, M.I. Friswell, Model updating in structural dynamics: a survey, J. Sound Vibr. 167 (1993) $347-375$ 
[3] L.M. Zhang, An overview of major development and issues in modal identification, In IMAC XXII: A Conference and Exposition on Structural Dynamics, 2004.

[4] W. Favoreel, B. De Moor, P. Van Overschee, Subspace state space system identification for industrial processes, J. Process Control 10 (2000) 149-155

[5] P. Van Overschee, B. DeMoor, Subspace Identification of Linear Systems: Theory, Implementation, Applications, Kluwer Academic Publishers, 1996

[6] M. Viberg, B. Wahlberg, B. Ottersten, Analysis of state space system identification methods based on instrumental variables and subspace fitting, Automatica 33 (1997) $1603-1616$

[7] A. Lee Swindlehurst, B. Ottersten, R. Roy, T. Kailath. A subspace fitting method for identification of linear statespace models, IEEE Trans. Automatic Control 40 (1995) $311-316$
[8] R. Serra, C. Gontier, M. Raffy, A subspace fitting method for structural modal identification in time domain, In ISMA 25: International Conference on Noise and Vibration Engineering, 2000

[9] A. Lee Swindlehurst, B. Ottersten, T. Kailath R. Roy, Multiple invariance esprit, IEEE Trans. Signal Process. 40 (1992) 867-881

[10] G.H. Golub, V. Pereyra, The differentiation of pseudoinverses and nonlinear least squares problems whose variables separate, SIAM J, 10 (1973) 413-432

[11] M.I. Friswell, J.E.T. Penny, Crack modeling for structural health monitoring, Structural Health Monitoring 1 (2002) $139-148$

[12] M. Lalanne, G. Ferraris, Rotordynamics Prediction in Engineering, Wiley, 1990 